\title{
Effect of Biologic Agents on Non-PASI Outcomes in Moderate-to-Severe Plaque Psoriasis: Systematic Review and Meta-Analyses
}

Erica L. Baker • Craig I. Coleman • Kurt M. Reinhart • Olivia J. Phung • Lisa Kugelman • Wendy Chen •

C. Michael White $\cdot$ Carla M. Mamolo $\cdot$ Joseph C. Cappelleri $\cdot$ William L. Baker

To view enhanced content go to www.dermtherapy-open.com Received: April 19, 2012 / Published online: June 19, 2012

(C) The Author(s) 2012. This article is published with open access at Springerlink.com

\section{ABSTRACT}

Introduction: The objective of this review was to conduct a systematic review with metaanalysis and Bayesian mixed treatment comparisons (MTC) evaluating the impact of biologics on non-Psoriasis Area and Severity Index (PASI) health outcomes in patients with moderate-to-severe plaque psoriasis.

Methods: MEDLINE and Cochrane Central Register of Controlled Trials were searched

E. L. Baker - C. I. Coleman - K. M. Reinhart .

O. J. Phung · L. Kugelman · W. Chen ·

C. M. White - W. L. Baker ( $\square)$

Health Outcomes, Policy and Economics (HOPE)

Collaborative Group of the University of

Connecticut School of Pharmacy and Hartford

Hospital, Hartford, CT, USA

e-mail: wbaker@uchc.edu

C. M. Mamolo

Pfizer Inc., Outcomes Research, Groton, CT, USA

J. C. Cappelleri

Pfizer Inc., Statistics, Groton, CT, USA

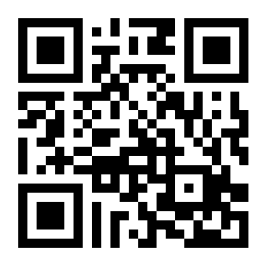

Enhanced content for this article is available on the journal web site: www.dermtherapy-open.com from 1966 to May 2009. Citations were screened for randomized, controlled trials of biologics versus either placebo or each other in adults with moderate-to-severe plaque psoriasis and reported any of several outcomes. Traditional and Bayesian MTC meta-analyses were conducted for each endpoint using either a random- or fixed-effect model where appropriate.

Results: Thirty-eight studies met eligibility criteria. All biologics showed significant improvement in achieving a good response on the static physician's global assessment (PGA) versus placebo while, in the MTC, differences were noted between individual drugs. In achieving a good response on the dynamic PGA, all biologics showed significant improvements over placebo, while the MTC showed significant improvements with the antiinterleukins versus anti-T cells. Relative to placebo, antitumor necrosis factor (TNF) agents and anti-interleukins showed significant improvements in the Dermatology Life Quality Index (DLQI). Compared with placebo, the anti-TNF agents showed significant improvements in both 36-item Medical Outcomes Study Short-Form General Health Survey (SF-36) mental and physical 
component scores, while anti-T cell agents showed no improvements. The MTC showed no differences between any biologics for either the DLQI or SF-36.

Conclusion: Individual biologics and classes showed consistent benefits across non-PASI health outcomes in patients with moderate-tosevere plaque psoriasis while MTC metaanalyses suggested that some differences exist.

Keywords: Biologics; Meta-analysis; Plaque psoriasis

\section{INTRODUCTION}

Psoriasis is a chronic inflammatory disorder seen in approximately $2-3 \%$ of the world's population, affecting the skin and often joints. The most common form is plaque psoriasis, which appears as sharply demarcated, erythematous areas covered with silvery-white scale $[1,2]$. The formation of psoriatic plaques involves the interplay of $\mathrm{T}$ cells, cytokines, and keratinocytes. The presence of activated $\mathrm{T}$ cells within psoriatic plaques and the response to $\mathrm{T}$ cell-directed therapy suggest an immunologic nature of the disease $[3,4]$. Various cytokines, such as tumor necrosis factor alpha (TNF $\alpha)$, are also present in psoriatic lesions, and may be a target for drug therapy [5]. Both cytokines and activated $\mathrm{T}$ cells promote the dysregulated growth of keratinocytes, leading to patches of erythematous, scaly skin.

Although there is no cure, treatment is directed at decreasing the signs and symptoms of psoriasis and modifying the natural progression of the disease. Methotrexate and cyclosporine are systemic agents that have proven efficacy but are limited by various toxicities including liver and kidney complications [1]. Numerous other systemic biologic agents are available and are categorized into three classes: anti-T cell agents (efalizumab, which was removed from the United States market in 2009, and alefacept), anti-TNF agents (infliximab, adalimumab, and etanercept), and antiinterleukin (IL)-12/23 agents (ustekinumab and an investigational agent briakinumab) [6].

Prior meta-analyses have demonstrated the benefits of these agents on various outcomes in moderate-to-severe plaque psoriasis $[7,8]$. This includes studies of the Psoriasis Area and Severity Index (PASI), a traditionally reported endpoint in this area [8]. However, none have comprehensively evaluated the impact of biologics on the physician's global assessment (PGA) or assessments of health-related quality of life (HRQoL). Thus, the authors conducted a systematic review with meta-analysis and Bayesian mixed treatment comparisons (MTC) evaluating the impact of biologics on health outcomes, including the PGA and patientreported HRQoL in patients with moderate-tosevere plaque psoriasis.

\section{METHODS}

\section{Search Strategy}

Two independent investigators conducted systematic literature searches of MEDLINE (1966 to May 2009) using the Cochrane Highly Sensitive and specific Search Strategy (Sensitivity and Precision Maximizing Version 2008) [9], and the Cochrane Central Register of Controlled Trials (1966 to May 2009). The following Medical Subject Heading and text keywords were used: psoriasis, plaque psoriasis, etanercept, infliximab, adalimumab, efalizumab, alefacept, ustekinumab, ABT-874 (briakinumab), $\mathrm{T}$ cell modulator, monoclonal 
antibody, tumor necrosis factor, biologic agent, and biologics.

\section{Study Selection}

Studies were included in the evaluation if they were (1) randomized, controlled trials (RCTs) of biologic agents to treat psoriasis versus placebo or each other; (2) conducted in adult patients with moderate-to-severe plaque psoriasis, usually defined as having an inadequate response to topical treatments alone and either having received prior systemic therapy or are candidates for such therapy; and (3) studies that reported efficacy data on clinical or humanistic outcomes. A Preferred Reporting Items for Systematic Reviews and MetaAnalyses (PRISMA) diagram was constructed for the literature search and selection process to describe the number of citations identified, studies excluded, and studies ultimately included (Fig. 1) [10].

\section{Validity Assessment}

All studies were reviewed and evaluated by two reviewers with disagreement resolved by discussion. The validated Jadad scale was used to assess the methodological quality of included trials [11]. This rating scale assesses inherent controllers of bias by using the following quality assessment criteria: use of and methods for generating randomization; use of and methods for double-blinding; and description of patient withdrawals and dropouts. One point was given for each satisfied criterion. An aggregate score between 0 and 5 was calculated for each included trial $(0=$ weakest, $5=$ strongest), with trials scoring $<3$ deemed to have lower methodological quality.

\section{Data Abstraction}

Through use of a standardized data abstraction tool, two reviewers independently collected data, with disagreement resolved through discussion or triage to a third reviewer. The following information was obtained from each trial: author identification, year of publication, study design and above-mentioned methodological quality criteria, source of study funding, study population, patient demographics, and co-morbidities.

\section{Study Endpoints}

The first endpoint is the PGA, which can be reported as either a static or dynamic scale [12]. There is no standard PGA, and different versions include six- or seven-point scales, which measure the severity of psoriasis. Terms such as "clear" or "excellent" (scores of 0 or 1) are used to define the clearing of psoriatic plaques from the skin, with higher scores denoting more severe disease. To measure HRQoL, two scales are used. The first is the (acute or chronic version) 36-item Medical Outcomes Study Short-Form General Health Survey (SF-36). It measures eight domains of HRQoL (physical function, social function, pain, physical and emotional role limitation, vitality, personal perceptions of health, and emotional well-being). Normal scores have a mean of 50, with higher scores being more favorable. The second is the Dermatology Life Quality Index (DLQI), a 10-item questionnaire that assesses the impact of chronic skin conditions on HRQoL, and is frequently used in clinical trials of psoriasis [13]. Scores range from 0 to 30, with 0 representing no disease impact on HRQoL. 


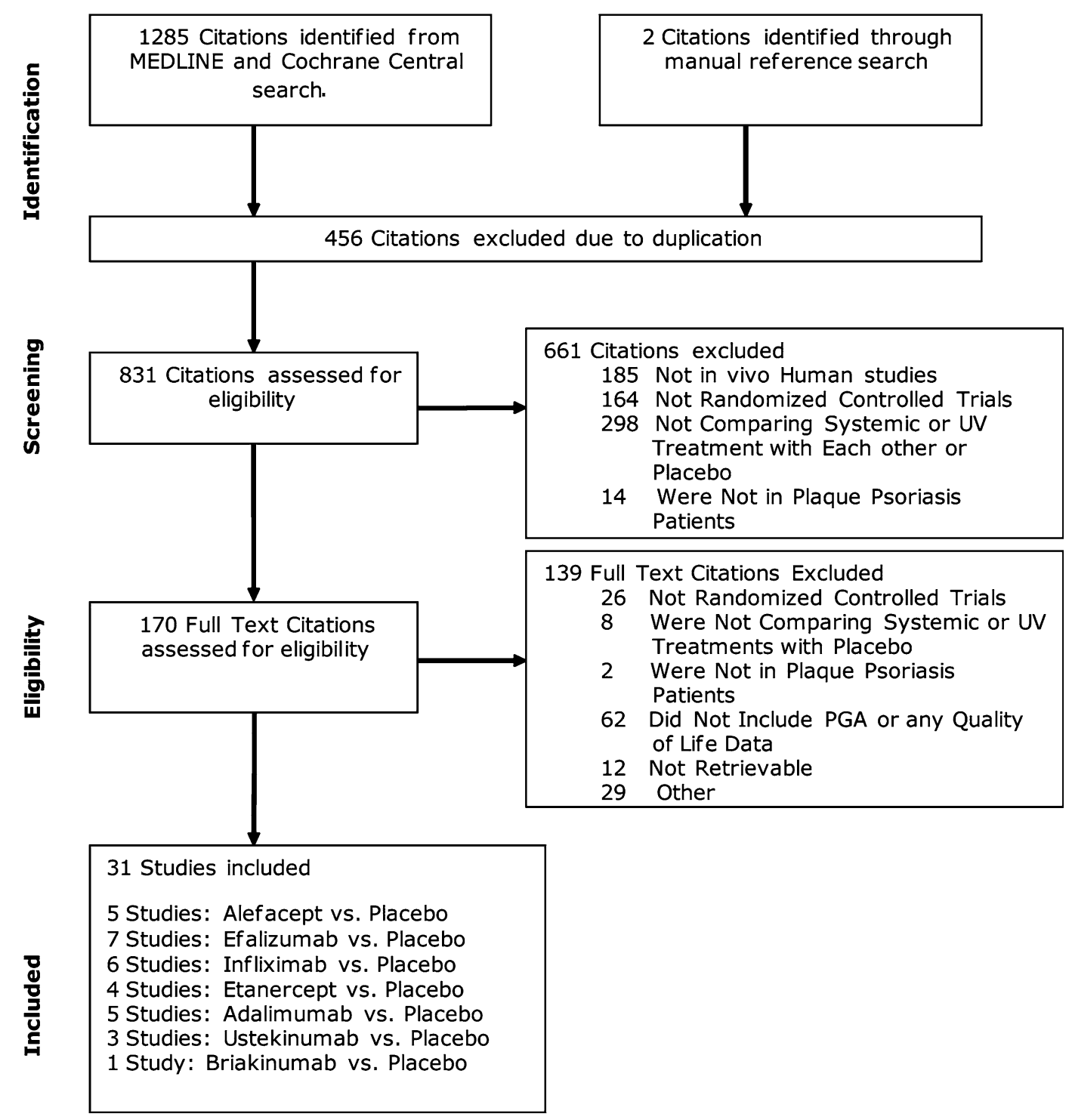

Fig. 1 Flow diagram of study selection. $P G A$ physician's global assessment

\section{Statistical Analysis}

Traditional meta-analysis was initially performed. For the primary analyses, only the US Food and Drug Administration (FDA)approved doses for each agent were included (for briakinumab, the investigational doses were included). In an attempt to avoid doublecounting individual agents in the analyses, when studies investigated more than one FDAapproved dose, only the highest dose was included in an analysis. This rule was not applied to either ustekinumab or briakinumab, which were not FDA-approved at the time this protocol was developed. Sensitivity analyses were also performed whereby data from all studies were included, regardless of dose. For dichotomous endpoints, weighted averages were reported as odds ratios (ORs) with associated 95\% CIs using a DerSimonian and Laird random-effects model [14]. For traditional meta-analysis for continuous outcomes, 
weighted averages were reported using a difference between means, with associated 95\% CIs using a DerSimonian and Laird random-effects model [14].

Statistical heterogeneity was addressed using the $\mathrm{I}^{2}$ statistic, which assesses the degree of inconsistency across studies and ranges from $0 \%$ to $100 \%$ with the higher percentage representing a higher likelihood of the existence of heterogeneity [15]. Visual inspection of funnel plots and Egger's weighted regression statistics were used to assess for the presence of publication bias [16, 17]. Statistics were performed using StatsDirect statistical software, version 2.4.6 (StatsDirect Ltd., Cheshire, UK) and Comprehensive Meta-Analysis, Version 2 (Biostat, Englewood, NJ, USA). A $P$ value of $<0.05$ was considered statistically significant for all analyses.

In addition to traditional meta-analysis, a MTC meta-analysis was conducted using previously validated WinBUGS code [18-22]. MTC methods were used to compare the different biologic agents to treat plaque psoriasis. These methods are a generalization of meta-analysis methods because they allow comparisons of agents not addressed within any of the individual trials. A random-effects model was fitted, taking into account the correlation structure induced by multi-arm trials. All MTC analyses were conducted using a Bayesian Markov chain Monte Carlo method and fitted in the freely availably Bayesian Software, WinBUGS.

\section{RESULTS}

\section{Literature Search}

A total of 1,287 citations were identified through the MEDLINE, Cochrane Central, and manual reference searches (Fig. 1). Of these, 31 studies were identified describing the following comparisons (Table 1) [23-53]: alefacept versus placebo $(n=5)$ [23-27]; efalizumab versus placebo $(n=7)$ [28-34]; infliximab versus placebo $(n=6)$ [35-40]; adalimumab versus placebo $(n=5)$ [4145]; etanercept versus placebo $(n=4)$ [46-49]; ustekinumab versus placebo $(n=3)$ [50, 51]; briakinumab versus placebo $(n=1)$ [53]. A total of 20 studies were included in the statistical analyses and evaluated FDA-approved doses: alefacept $(n=2)$ [26, 27], efalizumab $(n=4)$ [31-34], infliximab $(n=3) \quad$ [36-38], adalimumab $(n=4)[41,42,44,45]$, etanercept $(n=3)$ [46-48], ustekinumab $(n=3)$ [50-52], briakinumab $(n=1)$ [53].

\section{PGA Static Response Rate}

Sixteen RCTs evaluating seven drugs from three classes reported data on the PGA response rate using a static scale (Table 2) [23, 24, 27, 31, 33, $34,36,38,42,45-49,51-53]$. All of the RCTs were of high quality (Jadad $\geq 4$ ) and ranged from 8 to 24 weeks in duration. Ten studies [31, 33, $34,36,38,42,45,51-53$ ] defined their endpoint using a scale of "clear" or "minimal" rating while the remaining six studies $[23,24,27,46$, $48,49]$ used a scale of "clear" or "almost clear."

Six RCTs evaluated the anti-T cell agents with a single alefacept study [27] and three efalizumab studies [31, 33, 34] reporting results using FDA-approved doses. Seven RCTs evaluated the anti-TNF agents with two infliximab studies [36, 38], two adalimumab studies [42, 45], and two etanercept studies $[46,48]$ evaluating FDA-approved doses. Three RCTs evaluating the anti-IL-12/23 agents were included. Two ustekinumab studies [51, 52] reported results using the FDA-approved dose and the maximally effective dose of 
Table 1 Included study characteristics

\begin{tabular}{|c|c|c|c|c|c|c|}
\hline Study, ref & Years & $\begin{array}{l}\text { Study duration } \\
\text { (weeks) }\end{array}$ & Comparison & $N$ & Baseline PASI & $\begin{array}{l}\text { Jadad } \\
\text { Score }\end{array}$ \\
\hline \multicolumn{7}{|l|}{ Alefacept } \\
\hline \multirow[t]{4}{*}{ Ellis et al. [23] } & 2001 & 12 & ALA $0.025 \mathrm{mg} / \mathrm{kg} \mathrm{IV} \mathrm{QW}$ & 57 & $14(4-45)^{\mathrm{a}}$ & 5 \\
\hline & & & ALA 0.075 mg/kg IV QW & 55 & $15(4-45)^{\mathrm{a}}$ & \\
\hline & & & ALA $0.150 \mathrm{mg} / \mathrm{kg} \mathrm{IV} \mathrm{QW}$ & 58 & $20(7-33)^{\mathrm{a}}$ & \\
\hline & & & Placebo & 59 & $15(3-72)^{\mathrm{a}}$ & \\
\hline Gordon et al. [24] & 2003 & 12 & ALA $7.5 \mathrm{mg}$ IV QW & 367 & $14.4-15.9$ & 5 \\
\hline Feldman et al. [25] & 2004 & & Placebo & 186 & 15.1 & \\
\hline Finlayet al. [26] & 2003 & 12 & ALA 10 mg IM QW & 173 & $15.1(3.4-58.8)$ & 5 \\
\hline \multirow[t]{2}{*}{ Lebwohlet al. [27] } & 2003 & 14 & ALA 15 mg IM QW & 168 & $13.2(3.7-52.8)$ & \\
\hline & & & Placebo & 168 & $14.3(5.3-44.8)$ & \\
\hline \multicolumn{7}{|l|}{ Efalizumab } \\
\hline \multirow[t]{3}{*}{ Papp et al. [28] } & 2001 & 8 & EFA $0.1 \mathrm{mg} / \mathrm{kg}$ IV QW & 22 & $18.2 \pm 6.7$ & 4 \\
\hline & & & EFA $0.3 \mathrm{mg} / \mathrm{kg}$ IV QW & 75 & $19.1 \pm 7.3$ & \\
\hline & & & Placebo & 48 & $16.2 \pm 4.4$ & \\
\hline Gordon et al. [29] & 2003 & 12 & EFA $1 \mathrm{mg} / \mathrm{kg}$ SQ QW & 369 & $19.4(10.1-58.7)^{\mathrm{a}}$ & 4 \\
\hline Menter et al. [30] & 2005 & & Placebo & 187 & $19.4(11.4-50.3)^{\mathrm{a}}$ & \\
\hline \multirow[t]{3}{*}{ Leonardi et al. [31] } & 2005 & 12 & EFA $1 \mathrm{mg} / \mathrm{kg}$ SQ QW & 160 & $18.6(11.9-50.1)^{\mathrm{b}}$ & 5 \\
\hline & & & EFA $2 \mathrm{mg} / \mathrm{kg}$ SQ QW & 166 & $18.9(10-55.6)^{b}$ & \\
\hline & & & Placebo & 170 & $19.0(9.6-57.6)^{b}$ & \\
\hline Ortone et al. [32] & 2005 & 12 & EFA $1 \mathrm{mg} / \mathrm{kg}$ SQ QW & 529 & $23.6 \pm 9.7$ & 5 \\
\hline Dubertret et al. [33] & 2006 & & Placebo & 264 & $23.0 \pm 9.6$ & \\
\hline \multirow[t]{2}{*}{ Papp et al. [34] } & 2006 & 12 & EFA $1 \mathrm{mg} / \mathrm{kg}$ SQ QW & 450 & $19.1 \pm 7.5$ & 5 \\
\hline & & & Placebo & 236 & $18.7 \pm 7.0$ & \\
\hline \multicolumn{7}{|l|}{ Infliximab } \\
\hline \multirow[t]{3}{*}{ Chaudhari et al. [35] } & 2001 & 10 & INF $5 \mathrm{mg} / \mathrm{kg} \mathrm{IV}^{\mathrm{c}}$ & 11 & $22.1 \pm 11.5$ & 5 \\
\hline & & & INF $10 \mathrm{mg} / \mathrm{kg} \mathrm{IV}$ & 11 & $26.6 \pm 10.3$ & \\
\hline & & & Placebo & 11 & $20.3 \pm 5.5$ & \\
\hline Gottleib et al. [36] & 2004 & 10 & INF $3 \mathrm{mg} / \mathrm{kg} \mathrm{IV}$ & 99 & $20(15,26)^{\mathrm{d}}$ & 5 \\
\hline \multirow[t]{2}{*}{ Feldman et al. [37] } & 2005 & & INF $5 \mathrm{mg} / \mathrm{kg} \mathrm{IV}$ & 99 & $20(14,28)^{\mathrm{d}}$ & \\
\hline & & & Placebo & 51 & $18(15,27)^{\mathrm{d}}$ & \\
\hline \multirow[t]{2}{*}{ Reich et al. [38] } & 2005 & 24 & INF $5 \mathrm{mg} / \mathrm{kg} \mathrm{IV}$ & 301 & $22.9 \pm 9.3$ & 4 \\
\hline & & & Placebo & 77 & $22.8 \pm 8.7$ & \\
\hline
\end{tabular}


Table 1 continued

\begin{tabular}{|c|c|c|c|c|c|c|}
\hline Study, ref & Years & $\begin{array}{l}\text { Study duration } \\
\text { (weeks) }\end{array}$ & Comparison & $N$ & Baseline PASI & $\begin{array}{l}\text { Jadad } \\
\text { Score }\end{array}$ \\
\hline Menter et al. [39] & 2007 & 10 & INF $3 \mathrm{mg} / \mathrm{kg} \mathrm{IV}$ & 313 & $20.1 \pm 7.9$ & 5 \\
\hline \multirow[t]{2}{*}{ Feldman et al. [40] } & 2008 & & INF $5 \mathrm{mg} / \mathrm{kg} \mathrm{IV}$ & 314 & $20.4 \pm 7.5$ & \\
\hline & & & Placebo & 208 & $19.8 \pm 7.7$ & \\
\hline \multicolumn{7}{|l|}{ Adalimumab } \\
\hline Revicki et al. [41] & 2007 & 16 & ADA $40 \mathrm{mg}$ SQ QOW & 814 & $19.0 \pm 7.1$ & 5 \\
\hline Menter et al. [42] & 2008 & & Placebo & 397 & $18.8 \pm 7.1$ & \\
\hline \multirow[t]{3}{*}{ Shikiar et al. [43] } & 2007 & 12 & ADA $40 \mathrm{mg}$ SQ QOW & 45 & $16.7(5.4-39.0)^{\mathrm{a}}$ & 4 \\
\hline & & & ADA $40 \mathrm{mg}$ SQ QW & 50 & $14.5(2.3-42.4)^{\mathrm{a}}$ & \\
\hline & & & Placebo & 52 & $16.0(5.5-40.4)^{\mathrm{a}}$ & \\
\hline Revicki et al. [44] & 2008 & 16 & ADA $40 \mathrm{mg}$ SQ QOW & 110 & $19.4 \pm 7.4$ & 5 \\
\hline Saurat et al. [45] & 2008 & & Placebo & 53 & $19.2 \pm 6.9$ & \\
\hline \multicolumn{7}{|l|}{ Etanercept } \\
\hline \multirow[t]{4}{*}{ Leonardi et al. [46] } & 2003 & 12 & ETA $25 \mathrm{mg} \mathrm{SQ} \mathrm{QW}$ & 160 & $19.3 \pm 11.4$ & 4 \\
\hline & & & ETA 25 mg SQ BIW & 162 & $18.5 \pm 11.5$ & \\
\hline & & & ETA $50 \mathrm{mg}$ SQ BIW & 164 & $18.6 \pm 11.5$ & \\
\hline & & & Placebo & 166 & $18.4 \pm 11.6$ & \\
\hline Krueger et al. [47] & 2005 & 12 & ETA $25 \mathrm{mg}$ SQ BIW & 196 & $19.1 \pm 8.2$ & 5 \\
\hline \multirow[t]{2}{*}{ Papp et al. [48] } & 2005 & & ETA $50 \mathrm{mg}$ SQ BIW & 194 & $19.5 \pm 8.8$ & \\
\hline & & & Placebo & 193 & $18.6 \pm 8.6$ & \\
\hline \multirow{2}{*}{$\begin{array}{l}\text { Van de Kerkhof } \\
\text { et al. [49] }\end{array}$} & 2008 & 12 & ETA $50 \mathrm{mg}$ SQ QW & 96 & $21.4 \pm 9.3$ & 4 \\
\hline & & & Placebo & 46 & $21.0 \pm 8.7$ & \\
\hline \multicolumn{7}{|l|}{ Ustekinumab } \\
\hline \multirow[t]{5}{*}{ Krueger et al. [50] } & 2007 & 12 & UST $45 \mathrm{mg} \mathrm{SQ} \mathrm{X1}$ & 64 & $19.0 \pm 7.4$ & 4 \\
\hline & & & $\begin{array}{l}\text { UST } 45 \mathrm{mg} \\
\text { SQ QW X4 }\end{array}$ & 64 & $18.9 \pm 7.0$ & \\
\hline & & & UST 90 mg SQ X1 & 64 & $18.8 \pm 7.3$ & \\
\hline & & & UST $90 \mathrm{mg}$ SQ QW X4 & 64 & $19.0 \pm 7.9$ & \\
\hline & & & Placebo & 64 & $19.9 \pm 8.3$ & \\
\hline \multirow[t]{3}{*}{ Leonardi et al. [51] } & 2008 & 12 & UST $45 \mathrm{mg} \mathrm{SQ}{ }^{\mathrm{e}}$ & 255 & $20.5 \pm 8.6$ & 5 \\
\hline & & & UST $90 \mathrm{mg} \mathrm{SQ}$ & 256 & $19.7 \pm 7.6$ & \\
\hline & & & Placebo & 255 & $20.4 \pm 8.6$ & \\
\hline
\end{tabular}


Table 1 continued

\begin{tabular}{|c|c|c|c|c|c|c|}
\hline $\begin{array}{l}\text { Study, ref } \\
\text {. }\end{array}$ & Years & $\begin{array}{l}\text { Study duration } \\
\text { (weeks) }\end{array}$ & Comparison & $N$ & Baseline PASI & $\begin{array}{l}\text { Jadad } \\
\text { Score }\end{array}$ \\
\hline \multirow[t]{3}{*}{ Papp et al. [52] } & 2008 & 12 & UST $45 \mathrm{mg} \mathrm{SQ}{ }^{\mathrm{e}}$ & 409 & $19.4 \pm 6.8$ & 4 \\
\hline & & & UST $90 \mathrm{mg}$ SQ & 411 & $20.1 \pm 7.5$ & \\
\hline & & & Placebo & 410 & $19.4 \pm 7.5$ & \\
\hline \multicolumn{7}{|l|}{ Briakinumab } \\
\hline \multirow[t]{6}{*}{ Kimball et al. [53] } & 2008 & 12 & BRI $100 \mathrm{mg}$ SQ QOW & 30 & $20.0 \pm 6.9$ & 4 \\
\hline & & & BRI 200 mg SQ X1 & 30 & $18.0 \pm 6.7$ & \\
\hline & & & BRI 200 mg SQ QW X4 & 30 & $20.0 \pm 7.6$ & \\
\hline & & & BRI $200 \mathrm{mg} \mathrm{SQ} \mathrm{QOW}$ & 30 & $20.0 \pm 6.2$ & \\
\hline & & & BRI $200 \mathrm{mg} \mathrm{SQ}$ & 30 & $19.0 \pm 6.3$ & \\
\hline & & & QW X12 & 30 & $16.0 \pm 2.9$ & \\
\hline
\end{tabular}

$A D A$ adalimumab, $A L A$ alefacept, $B I W$ twice weekly, $B R I$ briakinumab, $E F A$ efalizumab, ET $A$ etanercept, $I N F$ infliximab, $I V$ intravenous, PASI psoriasis area and severity index, $Q W$ every week, $Q O W$ every other week, $S Q$ subcutaneous, $U S T$ ustekinumab

a Median (range)

b Mean (range)

c At weeks 0, 2, and 6

d Median (interquartile range)

e At weeks 0, 4, then every 12 weeks

briakinumab (200 mg every other week) was reported in another study [53].

Each individual agent, as well as each class, showed an increase in the odds of achieving a positive response (Fig. 2) [27, 31, 33, 34, 36, 38, $42,45,46,48,51-53]$. When all anti-T cell agent RCTs (OR 5.89, 95\% CI 4.34-7.99) and anti-TNF agent RCTs (OR 24.27, 95\% CI 15.66-37.61) were pooled, regardless of dose, slightly smaller overall effects were seen.

The MTC analysis included data from 13 trials of seven therapies in three drug classes that reported data on the PGA response rate using a static scale and included arms using the FDAapproved dose (Tables 3, 4) [27, 31, 33, 34, 36, 38, 42, 43, 46, 48, 51-53]. The placebo-based comparisons were similar to those discussed above, although generally had wider credible intervals (CrI). When the drug classes were analyzed, both the anti-TNF agents (OR 6.19, 95\% CrI 2.75-12.87) and anti-IL-12/23 agents (OR 7.60, 95\% CrI 3.25-18.80) were suggested to be superior to the anti-T cell agents. Pair-wise drug comparisons followed similar trends with many anti-TNF and anti-IL-12/23 agents showing superior results to the anti-T cell agents.

\section{PGA Dynamic Response Rate}

Seven RCTs evaluating three drugs from three classes reported data on the PGA response rate using a dynamic scale (Table 2) [28, 29, 31, 33, $35,39,50]$. All of the RCTs were of high quality (Jadad $\geq 4$ ) and ranged from 10 to 12 weeks in duration. All of the studies defined their endpoint using a rating of "clear" or "excellent."

Efalizumab was the only anti-T cell agent that provided data on the PGA dynamic 


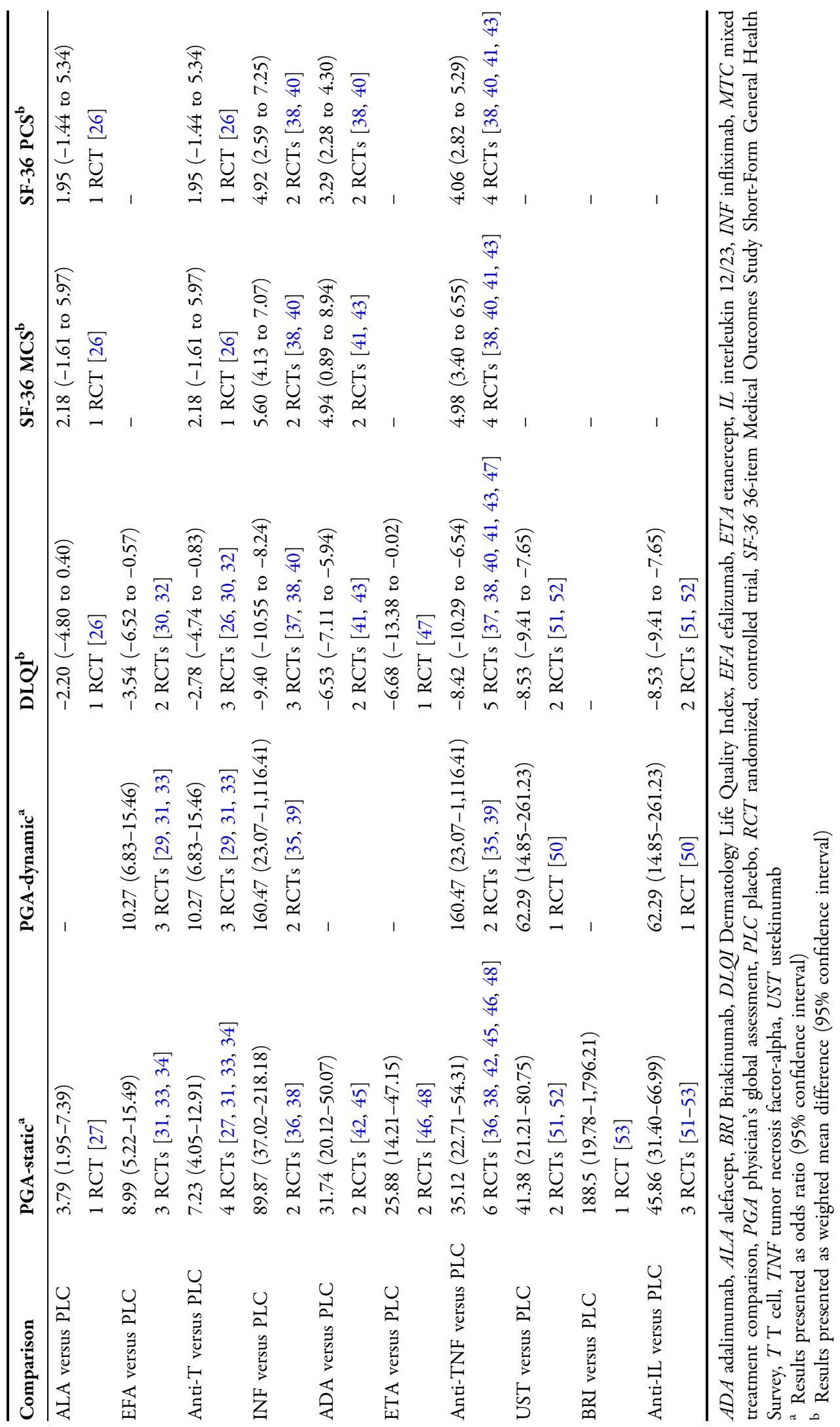




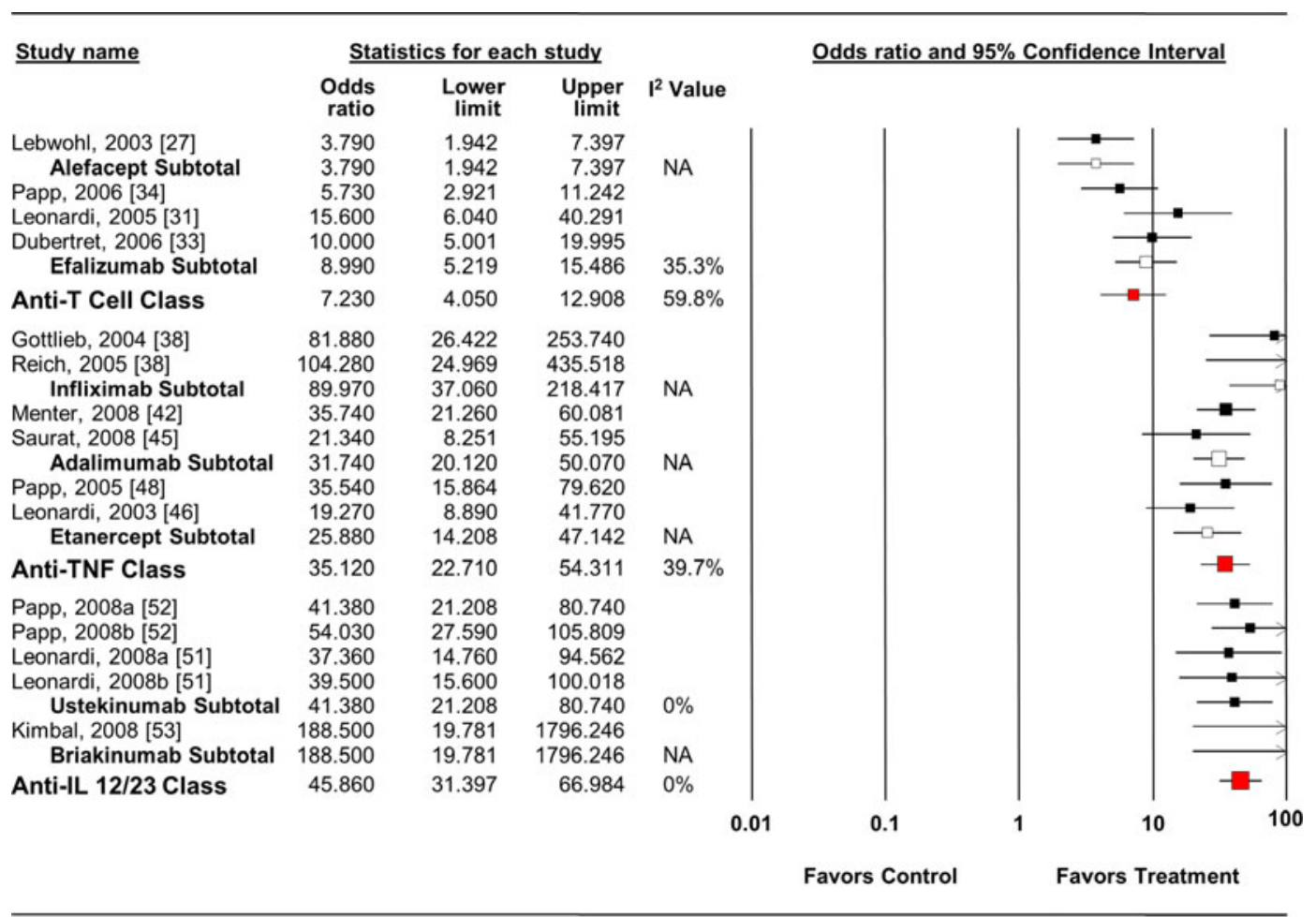

Meta Analysis

Fig. 2 Impact of biologic agents on static PGA response rate. IL interleukin, PGA physician's global assessment, $T N F$ tumor necrosis factor

endpoint with three RCTs evaluating FDA-approved doses [28, 29, 31, 33]. Infliximab was the only anti-TNF agent that reported data on this endpoint, with two RCTs evaluating FDA-approved doses $[35,39]$. Ustekinumab was the only anti-IL-12/23 agent that reported data on this endpoint [50].

Each individual agent, as well as each class, showed an increase in the odds of achieving a positive response (Fig. 3) [24, 31, 33, 35, 39, 50]. When all anti-T cell agent RCTs (OR 9.73, 95\% CI 6.54-14.49) and anti-TNF agent RCTs (OR 140.58, 95\% CI 39.14-504.97) were pooled, regardless of dose, similar overall effects were seen.

The MTC analysis included data from six RCTs of three therapies in three drug classes that reported data on the PGA response rate using a dynamic scale and included arms using the FDA-approved dose [29, 31, 33, 35, 39, 50]. Due to the small numbers of studies included in the analysis, many indirect comparisons yielded unreliable results (Tables 3,4 ). As a class, the anti-TNF agents were suggested to be superior to the anti-T cell agents (OR 22.53, 95\% CrI 2.61-206.3).

\section{Change in DLQI from Baseline}

Fifteen RCTs evaluating six drugs from three classes reported data on the change in DLQI score from baseline (Table 2) [23, 25, 26, 30, 32, 37, 38, $40,41,43,47,49-52]$. All of the RCTs were of high quality (Jadad $\geq 4$ ) and ranged from 10 to 24 weeks in duration. A lower score on the DLQI represents an improvement, with a score of 0 


\begin{tabular}{|c|c|c|c|c|c|c|c|c|c|c|c|c|c|c|c|c|c|c|c|c|c|c|c|c|}
\hline 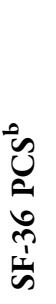 & 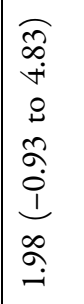 & & 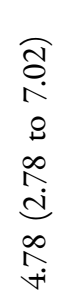 & 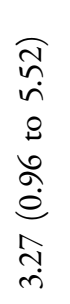 & 1 & 1 & 1 & 1 & 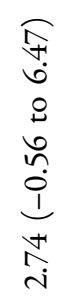 & 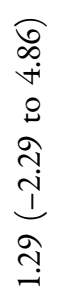 & 1 & 1 & 1 & 1 & 1 & 1 & 1 & 1 &  & 1 & 1 & 1 & 1 & 1 \\
\hline 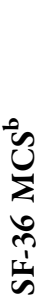 & 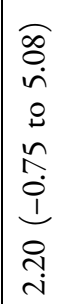 & & 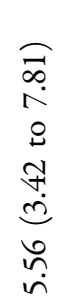 & $\begin{array}{l}\widehat{n} \\
\hat{n} \\
0 \\
\dot{b} \\
a \\
\dot{d} \\
\dot{d} \\
\dot{+} \\
\dot{+}\end{array}$ & 1 & 1 & 1 & 1 & 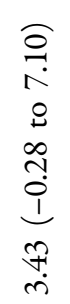 & 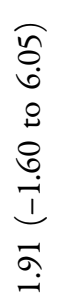 & 1 & 1 & 1 & 1 & 1 & 1 & 1 & I & $\begin{array}{l}\stackrel{+}{+} \\
\stackrel{+}{+} \\
\stackrel{+}{*} \\
\stackrel{\sim}{*}\end{array}$ & 1 & 1 & 1 & 1 & \\
\hline$\stackrel{\stackrel{\partial}{a}}{\stackrel{\theta}{a}}$ & 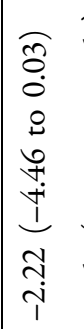 & 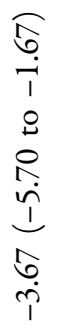 & 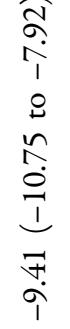 & $\begin{array}{l}\widehat{\sigma} \\
\stackrel{+}{+} \\
1 \\
0 \\
0 \\
0 \\
0 \\
0 \\
1 \\
1 \\
\hat{0} \\
\dot{0} \\
1\end{array}$ & $\begin{array}{l}0 \\
\hat{0} \\
i \\
i \\
0 \\
0 \\
\hat{N} \\
0 \\
i \\
1 \\
\hat{N} \\
0 \\
i\end{array}$ & 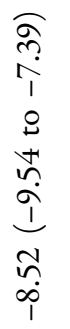 & & 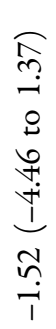 & 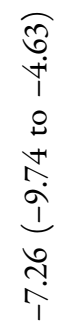 & 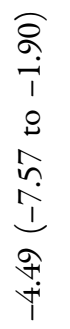 & 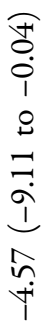 & 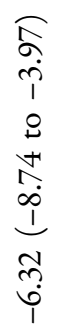 & & 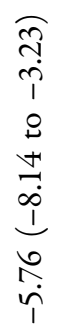 & 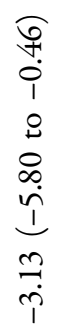 & 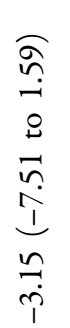 & 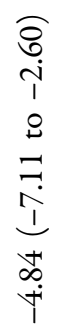 & 1 & 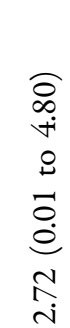 & 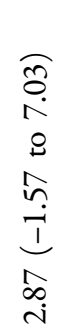 & 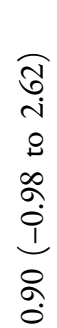 & & 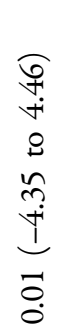 & 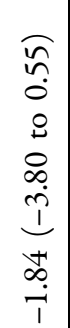 \\
\hline 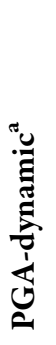 & 1 & 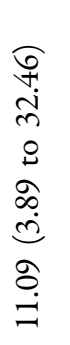 & 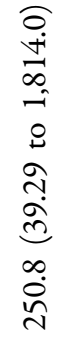 & 1 & 1 & 0 & 1 & 1 & 1 & 1 & 1 & 1 & 1 & 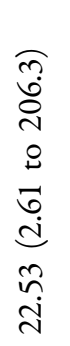 & 1 & 1 & $\tau$ & I & 1 & 1 & 0 & 1 & 1 & 1 \\
\hline 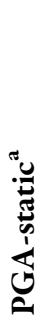 & 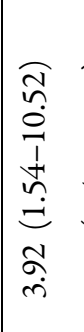 & $\begin{array}{l}\widehat{n} \\
\stackrel{n}{a} \\
\frac{1}{n} \\
\vec{n} \\
\vec{n} \\
\vec{a}\end{array}$ & 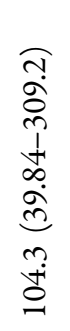 & 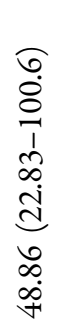 & 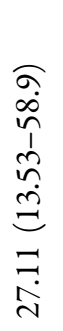 & 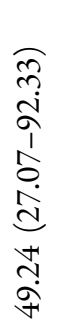 & 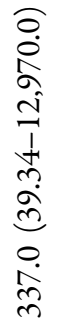 & 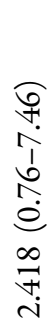 & 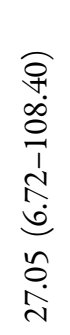 & 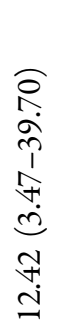 & 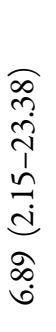 & 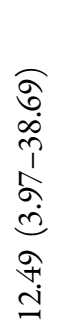 & 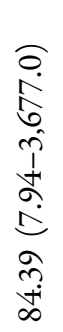 & 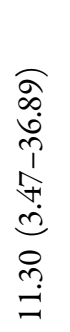 & 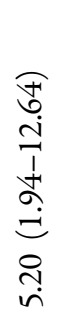 & 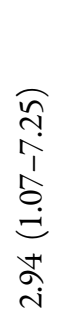 & 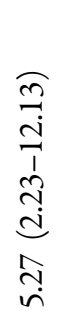 & 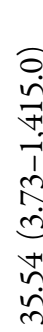 & 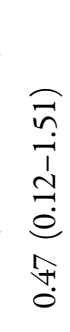 & 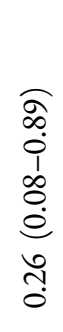 &  &  & 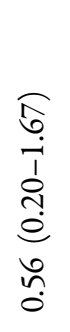 & 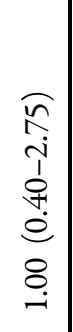 \\
\hline 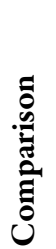 &  & 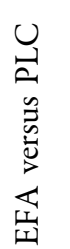 & 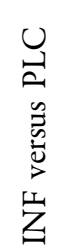 & 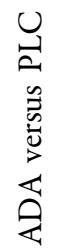 & 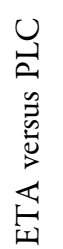 & 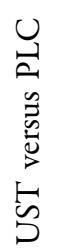 & 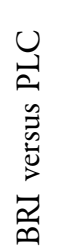 & 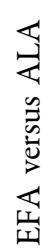 & 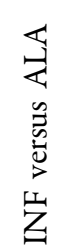 & 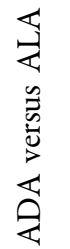 & 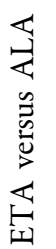 & 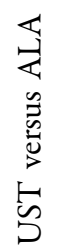 & 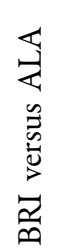 & 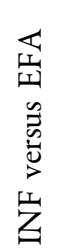 & 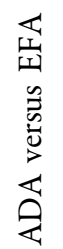 & 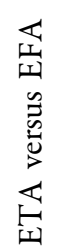 & 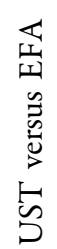 & 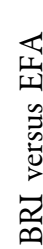 & 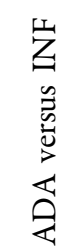 & 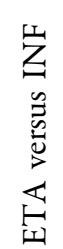 & 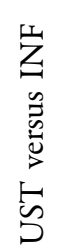 & 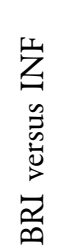 & 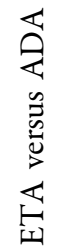 & 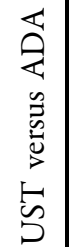 \\
\hline
\end{tabular}




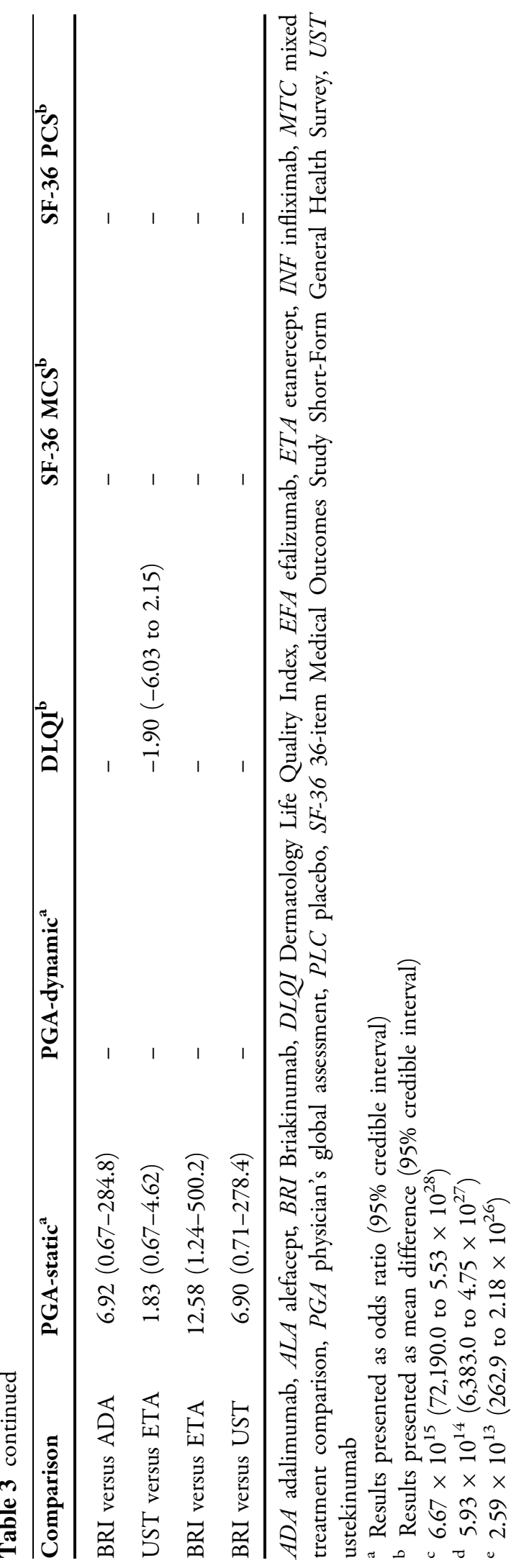

suggesting no impact of the disease on the patients' HRQoL scores.

Five RCTs evaluated the anti-T cell agents, with one alefacept study [26], and two efalizumab studies [30, 32] reporting results using FDA-approved doses. Seven RCTs evaluated the anti-TNF agents, with three infliximab studies [37, 38, 40], two adalimumab studies [41-43], and one etanercept study [47] reporting results using FDA-approved doses. Ustekinumab was the only anti-IL-12/23 agent that reported data on this endpoint with two studies reporting results using FDA-approved doses [50-52].

The anti-T cell agents as a class, as well as efalizumab alone significantly reduced the DLQI score from baseline (Fig. 4). No significant effect was seen with alefacept alone. Each individual anti-TNF agent, as well as the pooled class, significantly reduced the DLQI score from baseline. Similar effects were seen with ustekinumab. When all anti-T cell agent RCTs (WMD -2.377, 95\% CI -3.286 to -1.469), antiTNF agent RCTs (WMD $-8.03,95 \%$ CI -9.24 to -6.81), and anti-IL-12/23 RCTs (WMD -7.94, $95 \%$ CI -8.83 to -7.05 ) were pooled, regardless of dose, similar overall effects were seen.

The MTC analysis included data from seven RCTs of six therapies in three drug classes that reported data on the change from baseline in DLQI score and included arms using the FDAapproved dose (Tables 3, 4) [26, 30, 32, 37, 38, $40,41,43,47,51,52]$. As above, the placebocontrolled comparisons were similar between the MTC and traditional meta-analysis models. When the drug classes were compared, both the anti-TNF agents (mean difference $-7.77,95 \%$ CrI -8.27 to -3.23 ) and anti-IL-12/23 agents (mean difference $-5.37,95 \%$ CrI -7.89 to -2.82) reduced the DLQI to a greater extent than the anti-T cell agents. Many of the individual comparisons followed the same trend. 


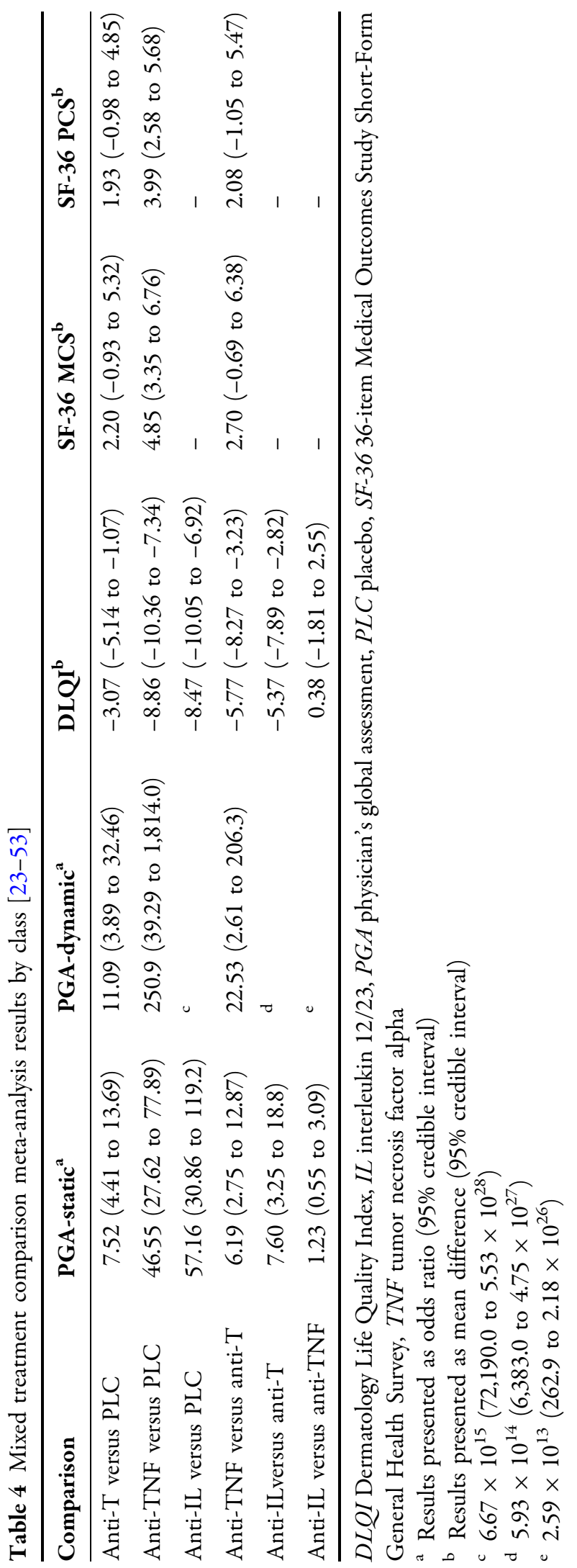

\section{Change in SF-36 from Baseline}

Eight RCTs evaluating four drugs from two classes reported data on the change in SF-36 score from baseline (Table 2) [23, 25, 26, 38, 40, $41,43,47]$. There were two component scores examined in this category, the physical and mental component summary (PCS and MCS) scores. All of the RCTs were of high quality (Jadad $\geq 4$ ) and ranged from 10 to 24 weeks in duration.

Alefacept was the only anti-T cell agent that reported data on these endpoints [23, 25, 26], with a single RCT [26] reporting results using FDA-approved doses. Five RCTs evaluated the anti-TNF agents, including infliximab [38-40], adalimumab [41, 43], and etanercept [47], all of which reported results using FDA-approved doses. No anti-IL-12/23 agent studies reported data on the SF-36 MCS or PCS.

Whereas alefacept had no significant impact on either the SF-36 MCS or PCS (Figs. 5, 6), each anti-TNF agent as well as the class significantly improved both SF-36 endpoints from baseline. When all anti-T cell agent RCTs $(\mathrm{MCS}=\mathrm{WMD}$ 2.18, 95\% CI -1.61 to 5.97; PCS $=$ WMD 1.95, $95 \% \mathrm{CI}-1.44$ to 5.34 ), and anti-TNF agent RCTs $(\mathrm{MCS}=\mathrm{WMD} \quad 4.56, \quad 95 \% \quad \mathrm{CI} \quad 3.59-5.54$; $\mathrm{PCS}=\mathrm{WMD} \quad 3.93, \quad 95 \%$ CI $3.09-4.78)$ were pooled, regardless of dose, similar overall effects were seen.

The MTC analysis included data from seven trials of three therapies in two drug classes that reported data on the change in SF-36 scores (both MCS and PCS) from baseline using the FDAapproved dose (Tables 3, 4) [23, 25, 26, 38, 40, $41,43]$. No differences between individual agents or drug classes were seen in the MTC model.

\section{Statistical Heterogeneity/Publication Bias}

Significant statistical heterogeneity was seen with the anti-T cell class for the static PGA 


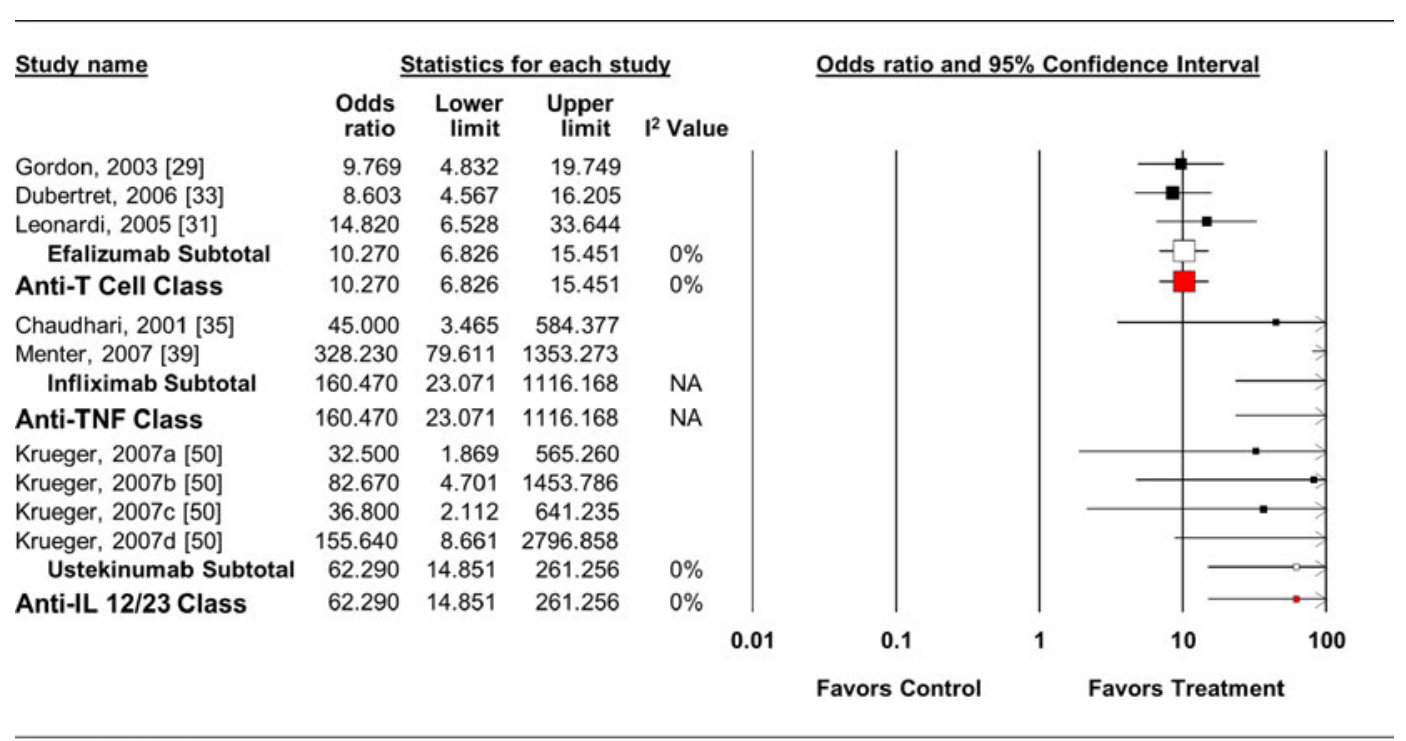

Meta Analysis

Fig. 3 Impact of biologic agents on dynamic PGA response rate. $I L$ interleukin, $P G A$ physician's global assessment, $T N F$ tumor necrosis factor

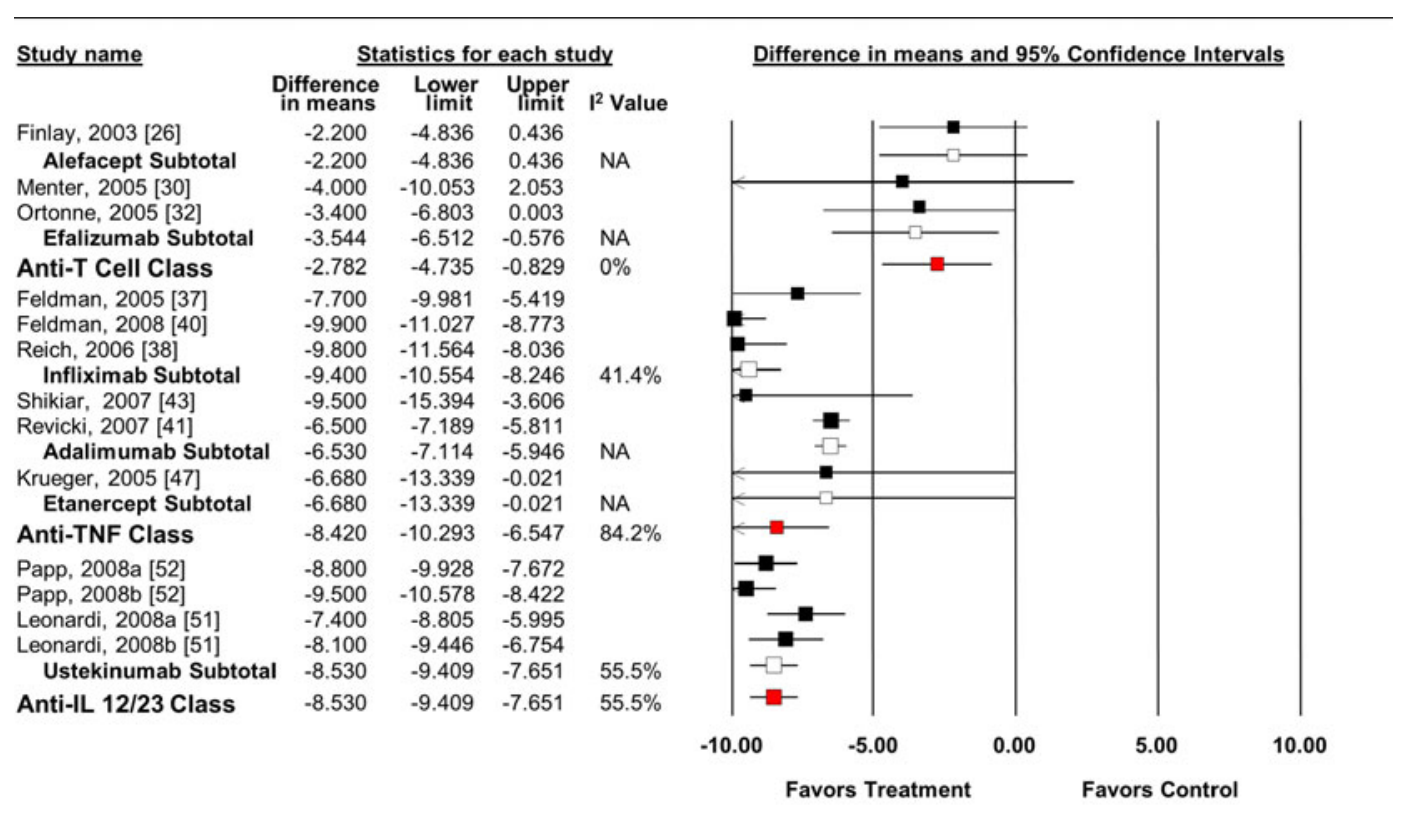

\section{Meta Analysis}

Fig. 4 Impact of biologic agents on change in DLQI from baseline. DLQI Dermatology Life Quality Index, IL interleukin, $T N F$ tumor necrosis factor

analysis $\left(\mathrm{I}^{2}=59.8 \%\right)$, and the anti-TNF class for the DLQI analysis $\left(\mathrm{I}^{2}=84.2 \%\right)$. In each case, the statistical heterogeneity was likely due to differences in the magnitude of effect rather than directionality. All other analyses had no significant heterogeneity $\left(\mathrm{I}^{2}<25 \%\right)$, 


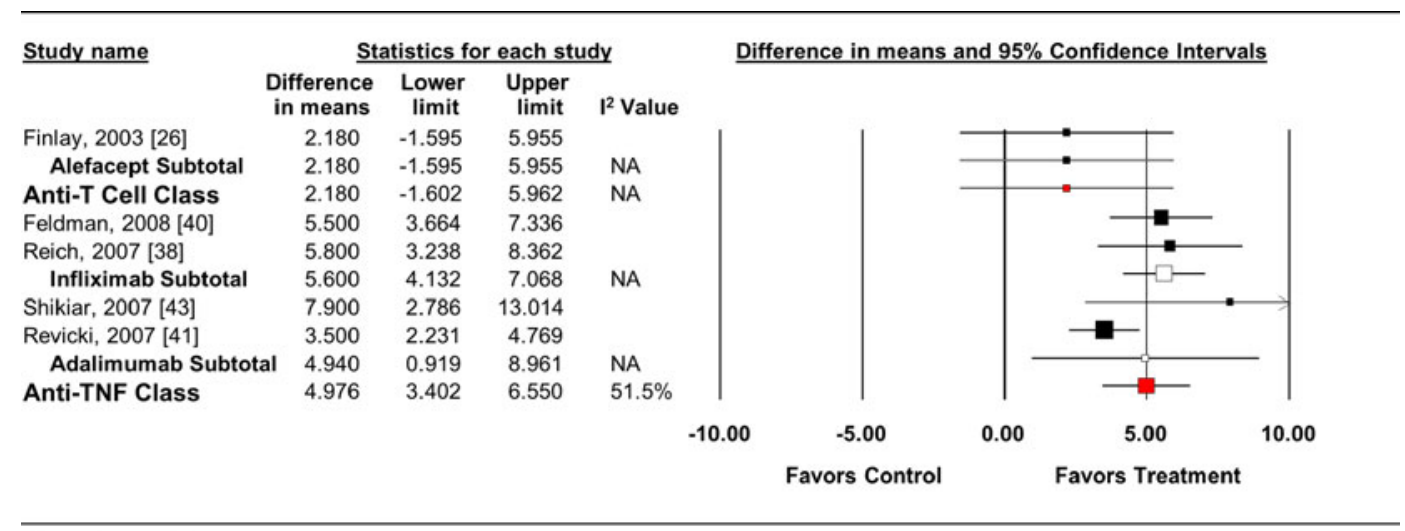

Meta Analysis

Fig. 5 Impact of biologic agents on change in SF-36 MCS from baseline. MCS mental component summary, SF-36 36-item Medical Outcomes Study Short-Form General Health Survey, TNF tumor necrosis factor

\begin{tabular}{|c|c|c|c|c|c|c|c|c|c|}
\hline \multirow[t]{2}{*}{$\underline{\text { Study name }}$} & \multicolumn{4}{|c|}{ Statistics for each study } & \multicolumn{5}{|c|}{ Difference in means and $95 \%$ Confidence Intervals } \\
\hline & $\begin{array}{l}\text { Difference } \\
\text { in means }\end{array}$ & $\begin{array}{l}\text { Lower } \\
\text { limit }\end{array}$ & $\begin{array}{c}\text { Upper } \\
\text { limit }\end{array}$ & $I^{2}$ Value & & & & & \\
\hline Finlay, 2003 [26] & 1.950 & -1.427 & 5.327 & & & | & & & \\
\hline Alefacept Subtotal & 1.950 & -1.427 & 5.327 & NA & & & & & \\
\hline Anti-T Cell Class & 1.950 & -1.433 & 5.333 & NA & & & & & \\
\hline Feldman, 2008 [40] & 3.900 & 2.423 & 5.377 & & & & & $\longrightarrow$ & \\
\hline Reich, 2007 [38] & 6.300 & 3.948 & 8.652 & & & & & - & \\
\hline Infliximab Subtotal & 4.920 & 2.593 & 7.247 & NA & & & & & \\
\hline Shikiar, 2007 [43] & 3.100 & -1.323 & 7.523 & & & & & & \\
\hline Revicki, 2007 [41] & 3.300 & 2.261 & 4.339 & & & & & - & \\
\hline Adalimumab Subtotal & 1 3.290 & 2.281 & 4.299 & NA & & & & $-\square-$ & \\
\hline \multirow[t]{3}{*}{ Anti-TNF Class } & 4.060 & 2.826 & 5.294 & $45.1 \%$ & & | & | & $\rightarrow-1$ & I \\
\hline & & & & & -10.00 & -5.00 & 0.00 & 5.00 & 10.00 \\
\hline & & & & & & Favors Control & & Favors Tre & \\
\hline
\end{tabular}

\section{Meta Analysis}

Fig. 6 Impact of biologic agents on change in SF-36 PCS from baseline. PCS physical component summary, SF-36 36-item Medical Outcomes Study Short-Form General Health Survey, TNF tumor necrosis factor

although many analyses had too few studies to formally test for its presence. Similarly, publication bias was not statistically significant in any analysis where enough studies were included for it to be tested (Egger's $P>0.05$ for all).

\section{DISCUSSION}

Overall, biologic agents were shown to be effective for improving clinical psoriasis symptoms (static and dynamic PGA), as well as measures of health-related quality of life (DLQI and SF-36). However, differences between drug classes and individual agents were seen using a MTC meta-analytic model. Although each individual agent and drug class showed significant improvements in the PGA (static and dynamic), the MTC model suggested that the anti-TNF agents and anti-IL 12/23 agents were both significantly better than the anti-T cell agents. Individual indirect drug comparisons showed similar trends. Each pharmacologic agent and drug class also showed significant improvements in the DLQI from baseline with the MTC model showing 
similar results to the PGA analysis. Studies have established that at least a five-point change in the DLQI can be considered clinically significant, indicating patients are a little better or worse [13]. Larger changes are required for more pronounced clinical improvements. Significant differences between individual agents ranged from three to seven points, and between classes from five to six points. Thus it can be debated that the differences seen equate to minimal clinical improvements in DLQI from one agent or class to the next.

Although the PASI is the outcome most commonly reported in efficacy clinical trials for biologic agents in chronic plaque psoriasis, many regulatory agencies have stressed the importance of patient-related outcome measures for their approval process [54]. Since prior meta-analyses have reported on the comparative effectiveness of biologics in patients with plaque psoriasis using PASI [8], the authors felt it was important to concentrate on other outcomes of interest, including the PGA and various HRQoL measures. This makes the information from our analyses pertinent to both practicing clinicians as well as regulatory bodies making drug coverage decisions. Moreover, studies evaluating the association between improvements in PASI with patient-related outcomes have been inconsistent $[55,56]$, with more recent data showing only a mild correlation [56].

Traditional meta-analyses showed that the anti-T cell agents did not have an impact on the SF-36 whereas improvements in both the MCS and PCS were seen with the anti-TNF agents. No significant effects were seen in the MTC model. These results are all intriguing, especially given that the MTC model showed similar point estimates to traditional meta-analysis for the direct placebo comparisons. This suggests that the Bayesian models used were reliable giving higher credence to the indirect comparison results.
The traditional meta-analysis results from this review are similar to those of prior published reports $[7,8,57,58]$. Additionally, this meta-analysis is the only one to utilize Bayesian MTC methodologies to provide indirect-comparisons between agents in addition to classes on non-PASI endpoints. This allows us to estimate the comparative treatment effects of available biologics and potentially guide treatment decisions in the absence of direct studies. The prior metaanalysis by Reich and colleagues used a Bayesian hierarchical model and concluded that no differences between the biologic agents existed at 24 weeks using the PASI 50, 75, or 90 [57]. No indirect comparisons were made by class. When compared with placebo, their results suggest the largest benefit with infliximab and etanercept versus other agents such as efalizumab and alefacept on the PASI 75.

Current guidelines from the American Academy of Dermatology, published in 2008, state that either an anti-T cell or anti-TNF agent can be used when a biologic agent is indicated with no preference given to any particular agent [1]. More recently published guidelines from the Deutsche Dermatologische Gesellschaft and the Berufsverband Deutscher Dermatologen in 2011 suggest that the anti-TNF agents (particularly adalimumab or infliximab) or ustekinumab should be the biologics of first choice in patients with psoriasis [59]. The choice of which anti-TNF agent to use should be patient specific and based on clinical need. The recommendations state that patients with stable chronic plaque psoriasis could consider etanercept or adalimumab as first choice due to their ease of administration (self subcutaneous injection). Because of the relatively new status of the anti-IL 12/23 agents, particularly ustekinumab, their use is recommended to be reserved as a second-line biologic agent if anti-TNF therapy has failed or cannot be used. Our results 
support these recommendations by showing that anti-TNF agents, as well as anti-IL 12/23 agents, significantly improve clinical efficacy (via the PGA) and HRQoL (via the DLQI) as compared with the anti-T cell agents in patients with moderate-to-severe plaque psoriasis. It is also worth noting that the choice of biologic of preference in some countries, such as the UK, incorporates both clinical as well as pharmacoeconomic considerations into their ranking of agents. The present study did not evaluate cost-effectiveness, and thus the authors cannot comment on this further.

The results of this meta-analysis must be interpreted cautiously as various limitations exist. Common limitations seen with traditional meta-analyses include heterogeneity and publication bias. Due to the low number of studies included in many of the analyses, statistical heterogeneity and publication bias could not be determined. A few analyses included a moderate degree of heterogeneity $\left(\mathrm{I}^{2}=50-75 \%\right)$. Often the differences seen were related to the magnitude of the effect rather than its direction. Thus, it is unlikely that this heterogeneity significantly altered the conclusions of this review. Differences in the included studies could have also contributed to both clinical as well as statistical heterogeneity. Studies varied by the severity of patients included (as measured by the baseline PASI score), duration of studies (ranging from 8 to 24 weeks), study quality (as assessed using the Jadad score), and inherent differences between the agents themselves. All of the studies included in this review were of high quality (Jadad $\geq 4$ ), and the inclusion of only the FDA-approved doses of the drugs into the primary analyses was done in an attempt to provide a somewhat homogeneous sample.

Similar to heterogeneity, publication bias could not be assessed in many analyses due to low study numbers. When it was available, publication bias was not likely due to an Egger's weighted regression statistic $P>0.05$. The systematic nature of this literature search, in addition to the relatively tight inclusion criteria, likely lead to the lack of publication bias.

Lastly, the short-term nature of many of the studies included in this review precludes extrapolation of our results to patients requiring long-term therapy. Although estimates from the MTC meta-analysis cannot simply be assumed accurate, were considered valid due, in part, to the similar results seen in the placebo-controlled comparisons between the MTC and traditional meta-analytic models.

Various knowledge gaps have been identified by this review. It is clear that comparative effectiveness studies evaluating the impact of biologics continue to be required. When these studies are designed and carried out it should be required that measures of HRQoL are collected and reported. In addition, comparative studies should be of a sufficient duration. As previously stated, most of the studies included in this review were 8-24 weeks in duration. Some included non-randomized 52-week extension studies that provided safety and efficacy data in an observational manner. Studies of a year or more in duration should maintain randomization in order to confirm whether differences seen between groups are seen over the long term.

\section{CONCLUSION}

Individual biologics and classes showed consistent benefits across health outcomes in patients with moderate-to-severe plaque psoriasis while MTC meta-analysis suggested that some differences exist. This work provides an important channel in the planning of future 
clinical trials aimed at defining the most efficacious biologic therapy.

\section{ACKNOWLEDGMENTS}

The authors acknowledge the contributions of Dr. Ajibade Ashaye, MBBS, MPH, and Mr. Jeffrey Mather, MS, to this project. This study was supported in part by a contract from Pfizer Inc. The sponsor provided input regarding the design of the study and review of the draft manuscript. E.L.B, C.I.C, K.M.R., O.J.P., L.K., W.C, C.M.W., W.L.B., and Dr. Ajibade Ashaye and Mr. Jeffrey Mather, were employees of Hartford Hospital, which received financial support from Pfizer in connection with the development of this manuscript. C.M.M. and J.C.C. are employed by Pfizer Inc. W.L.B. is the guarantor for this article, and takes responsibility for the integrity of the work as a whole.

Conflict of interest. C.M.M. and J.C.C. are employed by Pfizer Inc. No other authors report significant conflicts of interest germane to this project.

Open Access. This article is distributed under the terms of the Creative Commons Attribution Noncommercial License which permits any noncommercial use, distribution, and reproduction in any medium, provided the original author(s) and source are credited.

\section{REFERENCES}

1. Menter A, Gottlieb A, Feldman SR, et al. Guidelines of care for the management of psoriasis and psoriatic arthritis. J Am Acad Dermatol. 2008;58:826-60.

2. Nickoloff BJ, Nestle FO. Recent insights into the immunopathogenesis of psoriasis provide new therapeutic opportunities. J Clin Invest. 2004;113: 1664-75.

3. Panayi GS. Immunology of psoriasis and psoriatic arthritis. Bailliere's Clin Rheumatol. 1994;8:419-27.
4. Cheng JC, Smith LR, Froning KJ, et al. Persistence of T-cell clones in psoriatic lesions. Arch Dermatol. 1997;133:781-2.

5. Mease PJ, Goffe BS, Metz J, VanderStoep A, Finck B, Burge DJ. Etanercept in the treatment of psoriatic arthritis and psoriasis: a randomized trial. Lancet. 2000;356:385-90.

6. Nestle FO, Kaplan DH, Barker J. Psoriasis. N Engl J Med. 2009;361:496-509.

7. Woolacott $\mathrm{N}$, Hawkins $\mathrm{N}$, Mason $\mathrm{A}$, et al. Etanercept and efalizumab for the treatment of psoriasis: a systematic review. Health Technol Assess. 2006;10:1-233, i-iv.

8. Schmitt J, Zhang Z, Wozel G, et al. Efficacy and tolerability of biologic and nonbiologic systemic treatments for moderate-to-severe psoriasis: metaanalysis of randomized controlled trials. $\mathrm{Br} J$ Dermatol. 2008;159:513-26.

9. Higgins JPT, Green S (editors). Cochrane Handbook for Systematic Reviews of Interventions Version 5.0.1 [updated September 2008]. The Cochrane Collaboration, 2008. http://www.cochranehandbook.org. Accessed Dec 42008.

10. Liberati A, Altman DG, Tetzlaff J, et al. The PRISMA statement for reporting systematic reviews and meta-analyses of studies that evaluate health care interventions: explanation and elaboration. Ann Intern Med. 2009;151:W65-94.

11. Jadad AR, Moore A, Carroll D, et al. Assessing the quality of reports of randomized clinical trials: is blinding necessary? Controlled Clin Trials. 1996;17:1-12.

12. Feldman SR, Krueger GG. Psoriasis assessment tools in clinical trials. Ann Rheum Dis. 2005;64:ii65-8.

13. Khilji FA, Gonzalez M, Finlay AY. Clinical meaning of change in Dermatology Life Quality Index scores. Br J Dermatol. 2002;147(Suppl. 62):50.

14. Dersimonian R, Laird N. Meta-analysis in clinical trials. Control Clin Trials. 1986;7:177-88.

15. Higgins JP, Thompson SG, Deeks JJ, Altman DG. Measuring inconsistency in meta-analyses. BMJ. 2003;327:557-60.

16. Egger M, Davey Smith G, Schneider M, Minder C. Bias in meta-analysis detected by a simple, graphical test. BMJ. 1997;315:629-34.

17. Duval S, Tweedie R. Trim and fill: a simple funnelplot-based method of testing and adjusting for publication bias in meta-analysis. Biometrics. 2000;56:455-63. 
18. Lu G, Ades AF. Combination of direct and indirect evidence in mixed treatment comparisons. Stat Med. 2004;23:3105-24.

19. Caldwell DM, Ades AE, Higgins JP. Simultaneous comparison of multiple treatments: combining direct and indirect evidence. BMJ. 2005;331:897-900.

20. Cooper NJ, Sutton AJ, Lu G, Khunti K. Mixed comparison of stroke prevention treatments in individuals with nonrheumatic atrial fibrillation. Arch Intern Med. 2006;166:1269-75.

21. Coleman CI, Baker WL, Kluger J, White CM. Antihypertensive medication and their impact on cancer incidence: a mixed treatment comparison meta-analysis of randomized controlled trials. J Hypertens. 2008;26:622-9.

22. Baker WL, Baker EL, Coleman CI. Pharmacologic treatments for chronic obstructive pulmonary disease: a mixed-treatment comparison metaanalysis. Pharmacotherapy. 2009;29:891-905.

23. Ellis C, Krueger G, for the Alefacept Clinical Study Group. Treatment of chronic plaque psoriasis by selective targeting of memory effector T lymphocytes. N Eng J Med. 2001;345:248-55.

24. Gordon KB, Langley RA. Remittive effects of intramuscular alefacept in psoriasis. J Drugs Dermatol. 2003;2:624-8.

25. Feldman S, Menter A, Koo J. Improved healthrelated quality of life following a randomized controlled trial of alefacept treatment in patients with chronic plaque psoriasis. $\mathrm{Br} \mathrm{J}$ Dermatol. 2004;150:317-26.

26. Finlay A, Salek M, Haney J, for the Alefacept Clinical Study Group. Intramuscular Alefacept improves health-related quality of life in patients with chronic plaque psoriasis. Dermatology. 2003;206:307-15.

27. Lebwohl M, Christophers E, Langley R, Ortonne JP, Roberts J, Griffiths CEM. An international, randomized, double-blind, placebo-controlled phase 3 trials of intramuscular alefacept in patients with chronic plaque psoriasis. Arch Dermatol. 2003;139:719-27.

28. Papp K, Bissonnette $\mathrm{R}$, Krueger $\mathrm{J}$, et al. The treatment of moderate to severe psoriasis with a new anti-CD11a monoclonal antibody. J Am Acad Dermatol. 2001;45:665-74.

29. Gordon KB, Papp KA, Hamilton TK, et al. Efalizumab for patients with moderate to severe plaque psoriasis. JAMA. 2003;290:3073-80.

30. Menter A, Gordon K, Carey W, et al. Efficacy and safety observed during 24 weeks of efalizumab therapy in patients with moderate to severe plaque psoriasis. Arch Dermatol. 2005;141:31-8.

31. Leonardi CL, Papp KA, Gordon KB, et al. Extended efalizumab therapy improves chronic plaque psoriasis: results from a randomized phase III trial. J Am Acad Dermatol. 2005;52:425-33.

32. Ortonne J, Shear N, Shumack S, Henniger E, the Clear Multinational Study Group. Impact of efalizumab on patient-reported outcomes in highneed psoriasis patients: results of the international, randomized, placebo-controlled Phase III Clinical Experience Acquired with Raptiva (CLEAR) trial. BMC Dermatol. 2005;5:13.

33. Dubertret L, Sterry W, Bos J, et al. Clinical experience acquired with the efalizumab $\left(\right.$ Raptiva $\left.^{\circledR}\right)$ (CLEAR) trial in patients with moderate-to-severe plaque psoriasis: results from a phase III international randomized, placebocontrolled trial. Br J Dermatol. 2006;155:170-81.

34. Papp K, Bressinick R, Fretzin S, et al. Safety of efalizumab in adults with chronic moderate to severe plaque psoriasis: a phase IIb, randomized, controlled trial. Int J Dermatol. 2006;45:605-14.

35. Chadhari U, Romano P, Mulcahy LD, Dooler LT, Baker DG, Gottlieb AB. Efficacy and safety of infliximab monotherapy for plaque-type psoriasis: a randomized trial. Lancet. 2001;357:1842-7.

36. Gottlieb $\mathrm{AB}$, Evans $\mathrm{R}, \mathrm{Li} \mathrm{S}$, et al. Infliximab induction therapy for patients with severe plaquetype psoriasis: a randomized, double-blind, placebo-controlled trial. J Am Acad Dermatol. 2004;51:534-42.

37. Feldman S, Gordon $\mathrm{K}$, Bala $\mathrm{M}$, et al. Infliximab treatment results in significant improvement in the quality of life of patients with severe psoriasis: a double-blind placebo-controlled trial. $\mathrm{Br} \mathrm{J}$ Dermatol. 2005;152:954-60.

38. Reich K, Nestle FO, Papp $\mathrm{K}$, et al. Infliximab induction and maintenance therapy for moderateto-severe psoriasis: a phase III, multicentre, doubleblind trial. Lancet. 2005;366:1367-74.

39. Menter A, Feldman S, Weinstein G, et al. A randomized comparison of a continuous vs. intermittent infliximab maintenance regimens over 1 year in the treatment of moderate-to-severe plaque psoriasis. J Am Acad Dermatol. 2007; 56(31):e1-15.

40. Feldman S, Gottleib B, Bala $\mathrm{M}$, et al. Infliximab improves health-related quality of life in the presence of comorbidities among patients with moderate-to-severe psoriasis. $\mathrm{Br} \mathrm{J}$ Dermatol. 2008;159:704-10. 
41. Revicki D, Willian M, Menter A, et al. Impact of adalimumab from a phase III clinical trial in patients with moderate to severe plaque psoriasis. J Dermatol Treat. 2007;18:341-50.

42. Menter A, Tyring SK, Gordon K, et al. Adalimumab therapy for moderate to severe psoriasis: a randomized, controlled phase III trial. J Am Acad Dermatol. 2008;58:106-15.

43. Shikiar R, Heffernan M, Langley R, Willian M, Okun $\mathrm{M}$, Revicki D. Adalimumab treatment is associated with improvement in health-related quality of life in psoriasis: patient-reported outcomes from a phase II randomized controlled trial. J Dermatol Treat. 2007;18:25-31.

44. Revicki D, Willian M, Saurat J, et al. Impact of adalimumab treatment on health-related quality of life and other patient-reported outcomes: results from a 16-week randomized controlled trial in patients with moderate to severe psoriasis. $\mathrm{Br} \mathrm{J}$ Dermatol. 2008;158:549-57.

45. Saurat J, Stingl G, Dubertret L, et al. Efficacy and safety results from the randomized controlled comparative study of adalimumab vs. methotrexate vs. placebo in patients with psoriasis (CHAMPION). Br J Dermatol. 2008;158:558-66.

46. Leonardi CL, Powers JL, Matheson RT, et al. Etanercept as monotherapy in patients with psoriasis. N Engl J Med. 2003;349:2014-22.

47. Krueger G, Langley R, Finlay A, et al. Patientreported outcomes of psoriasis improvement with etanercept therapy: results of a randomized phase III trial. Br J Dermatol. 2005;153:1192-9.

48. Papp KA, Tyring S, Lahfa M, et al. A global phase III randomized controlled trial of etanercept in psoriasis: safety, efficacy, and effect of dose reduction. Br J Dermatol. 2005;152:1304-12.

49. Van de Kerkhof P, Segaert S, Lahfa M, et al. Once weekly administration of etanercept $50 \mathrm{mg}$ is efficacious and well tolerated in patients with moderate-to-severe plaque psoriasis: a randomized controlled trial with open-label extension. $\mathrm{Br} \mathrm{J}$ Dermatol. 2008;159:1177-85.

50. Krueger GG, Langley RG, Leonardi C, et al. A human interleukin-12/23 monoclonal antibody for the treatment of psoriasis. $\mathrm{N}$ Engl J Med. 2007;356:580-92.
51. Leonardi CL, Kimball AB, Papp KA, et al. Efficacy and safety of ustekinumab, a human interleukin$12 / 23$ monoclonal antibody, in patients with psoriasis: 76-week results from a randomized, double-blind, placebo-controlled trial (PHOENIX 1). Lancet. 2008;371:1665-74.

52. Papp KA, Langley RG, Lebwohl M, et al. Efficacy and safety of ustekinumab, a human interleukin$12 / 23$ monoclonal antibody, in patients with psoriasis: 52-week results from a randomized, double-blind, placebo controlled trial (PHOENIX 2). Lancet. $2008 ; 371: 1675-84$.

53. Kimball AB, Gordon KB, Langley RG, Menter A, Chartash EK, Valdes J. Safety and efficacy of ABT874 , a fully human interleukin $12 / 23$ monoclonal antibody, in the treatment of moderate to severe chronic plaque psoriasis. Arch Dermatol. 2008;144:200-7.

54. United States Food and Drug Administration. http://www.fda.gov/downloads/Drugs/GuidanceCom plianceRegulatoryInformation/Guidances/UCM1932 82.pdf. Accessed May 112012.

55. Revicki DA, Willian MK, Menter A, Saurat JH, Harnam N, Kaul M. Relationship between clinical response to therapy and health-related quality of life outcomes in patients with moderate to severe plaque psoriasis. Dermatology. 2008;216:260-70.

56. Schafer I, Hacker J, Rustenbach SJ, Radtke M, Franzke N, Augustin M. Concordance of the Psoriasis Area and Severity Index (PASI) and patient-related outcomes in psoriasis treatment. Eur J Dermatol. 2010;20:62-7.

57. Katugampola RP, Lewis VJ, Finaly AY. The Dermatology Life Quality Index: assessing the efficacy of biological therapies for psoriasis. Br J Dermatol. 2007;156:945-50.

58. Reich K, Sinclair R, Roberts G, Griffiths CEM, Tabberer M, Barker J. Comparative effects of biological therapies on the severity of skin symptoms and health-related quality of life in patients with plaque-type psoriasis: a metaanalysis. Curr Med Res Opin. 2008;24:1237-54.

59. Nast A, Boehncke WH, Mrowietz U, et al. German S3-guidelines on the treatment of psoriasis vulgaris (short version). Arch Dermatol Res. 2012;304: 87-113. 